\title{
Pan(proto)psychism and the Relative-State Interpretation of Quantum Mechanics*
}

\author{
Yu Feng ${ }^{\dagger 1}$ \\ ${ }^{1}$ Foundations of Quantum Mechanics Research Group
}

\section{Introduction}

The interpretation of quantum mechanics (QM) and the hard problem of consciousness are two puzzling problems that are seemingly separate. Among all the mainstream interpretations of QM, Everett's relative-state (RS) interpretation is perhaps the only realist view that preserves the simplicity of QM, despite being counter-intuitive. It has a counterpart, which is constitutive Russellian pan(proto)psychism (CRP). As a promising solution to the hard problem of consciousness, CRP also holds a simple but counter-intuitive view of realism.

It is possible that RS and CRP are in fact deeply related. They both realise some symmetry between micro- and macrosystems (distinguished in terms of the sizes of the physical systems under consideration). In particular, RS extends the quantum world, originally for microsystems only, to cover macrosystems. It claims that an observed result is not about the state of a microsystem alone, but instead about the state of a

${ }^{*}$ The author is grateful to David Chalmers and David Pearce (see physicalism.com for his own view) for inspiring this work and providing insightful comments, and to C.M. Stewart for proofreading.

Correspondence: foundationsofQM@gmail.com 
microsystem relative to the state of an observer, which is a macrosystem. CRP, on the other hand, extends phenomenal properties to microsystems, thus realising symmetry in the opposite direction. It is perhaps unwise to argue for the two proposals separately, as extra psychophysical laws might be required in either case. Arguing for both simultaneously, on the other hand, releases much theoretical burden, and may bind the two options into one that solves both problems, e.g. the interpretation of QM and the hard problem of consciousness.

The strategy is to show that CRP and RS are not only consistent, but also provide support to each other. Rejecting one entails rejection of the other. Therefore, if critics accuse one of them being counter-intuitive while deeming the other acceptable, they would hesitate to reject either.

\section{Arguments for Mutual Support}

\subsection{From CRP to RS}

We may first ask CRP to provide support to the RS interpretation of QM. First, let's define CRP properly. Phenomenal properties are the properties characterising what it is like to be something. Panpsychism holds that phenomenal properties are instantiated in some microsystems, e.g. fundamental particles, as well as microphysical properties. Such phenomenal properties are called microphenomenal properties, in contrast to the macrophenomenal properties that are instantiated in conscious beings like us. Panprotopsychism, on the other hand, suggests the instantiation of protophenomenal properties in microsystems, which are themselves not phenomenal, but in combination they constitute phenomenal properties. Russellian pan(proto)psychism is the thesis that microphenomenal or protophenomenal properties are the categorical bases (quiddities) of microphysical properties. Constitutive pan(proto)psychism is the thesis that all macrophenomenal truths are grounded in micro(proto)phenomenal truths. 
Now switch to quantum mechanics. If we examine alternative interpretations of QM, there are two general classes regarding the interpretation of quantum states (or wave functions). The anti-realist view that quantum states merely play an epistemic role leads us to the somehow instrumental Copenhagen interpretation and the more radical quantum Bayesianism (QBism). On the other side of the spectrum, treating quantum states as ontological entities leaves us with two options, depending on whether wave function collapse is ontological or merely epistemic. The former consists of various versions of collapse theories, and the latter consists of hidden variable theories and the RS interpretation. Collapse theorists insist that there is a physical collapse process which reduces quantum states to their classical counterparts. This includes objective collapse theories and the von Neumann-Wigner interpretation which argues that consciousness plays a role in the collapse process. On the other hand, hidden variable theorists claim that the quantum mechanical indeterminism is due to the incompleteness of the quantum-state description of reality. Collapse merely reflects our lack of knowledge (about the values of the hidden variables). Our strategy here is to combine CRP with each of these alternatives to demonstrate their incompatibility.

For epistemic interpretation of quantum states, we will take QBism as an example. It claims that quantum states are merely epistemic, reflecting the partial knowledge we have about reality. Collapse is therefore an inferential process (e.g. Bayesian inference) that reduces our belief from a partially informed quantum state to the fully informed classical counterpart. Metaphysically only classical entities exist and evolve according to the laws of classical physics. But then how do we explain the unitary evolution of wave function? If it is purely epistemic, it is arguably isomorphic to certain laws (or relations) that govern the dynamics of our macrophenomenal properties. According to CRP, macrophenomenal truths are grounded in micro(proto)phenomenal truths, which in turn ground microphysical truths categorically. Therefore, unitary evolution is likely to be isomorphic to certain truths (structural or relational) about microphysical properties. But if quantum states are merely epistemic, no microphysical relation is isomorphic to unitary evolution (or other relations in QM that are missing in classical physics). So the epistemic interpretation is arguably incompatible with CRP. To put this formally: 
1. If CRP is true, then macrophenomenal truths and microphysical truths are both grounded in micro(proto)phenomenal truths.

2. If quantum states are epistemic, then some macrophenomenal relations are isomorphic to unitary evolution (we simply say some macrophenomenal relations are unitary).

3. If some macrophenomenal relations are unitary, and macrophenomenal truths are grounded in micro(proto)phenomenal truths, then some micro(proto)phenomenal relations are unitary.

4. If some micro(proto)phenomenal relations are unitary and they ground certain microphysical relations, then the grounded microphysical relations are also unitary.

5. If quantum states are merely epistemic, no microphysical relation is unitary.

$\therefore 6$. If CRP is true, quantum states are not merely epistemic.

Proponents of the epistemic interpretation may reject point 2,3 or 4 . On point 2 they may argue that mathematical truths are epistemic but are probably not grounded in macrophenomenal truths. However, mathematical knowledge is not within our direct acquaintance. Instead, certain logical inference is required to acquire such knowledge. But when I let an electron evolve and then re-measure its state, no inference is explicitly needed in order to grasp the result. If the evolution merely goes on in my head, then it is reasonable to ask for its ground. On point 4, one might argue that CRP does not explicitly rule out the possibility that certain micro(proto)phenomenal relations do not ground microphysical relations. Even if this is possible, it introduces extra relations that are physically irrelevant. This is perhaps a very dualistic view of CRP and is ontologically costy. Besides, it faces the problem of epiphenomenalism. Perhaps the only viable objection is against point 3, arguing that unitary evolution is emergent upon the constitution of macrophenomenal properties. But this cannot be explained either empirically (our perception seems to match perfectly well with what the laws of classical physics dictate) or evolutionarily (having QM laws embedded does not improve our adaptability and is not an economic evolutionary strategy).

The von Neumann-Wigner interpretation holds a view of realism for both quantum states and their collapse. It further claims that consciousness plays a causal role in the col- 
lapse process. This means that macrophenomenal properties trigger collapse. If we accept CRP, then macrophenomenal properties can be reduced to micro(proto)phenomenal properties (plus some physical properties). However, not all combinations of micro(proto)phenomenal properties constitute macrophenomenal properties. For example, no macrophenomenal property is instantiated in a two-electron system that does not necessitate collapse under the von Neumann-Wigner interpretation, even if there are micro(proto)phenomenal properties instantiated in each electron. Therefore only certain collections of micro(proto)phenomenal properties trigger collapse. But collapse, commonly regarded as a fundamental process, is arguably not strongly emergent. This is more clear if we take a panpsychist view: either none of the phenomenal properties would necessitate collapse, or any of the phenomenal properties would necessitate collapse. But if the latter is true, collapse is universal, contradicting the facts about QM and the von Neumann-Wigner interpretation itself. To put this formally:

1. If CRP is true, then macrophenomenal properties are constituted by some (but not all) combinations of micro(proto)phenomenal properties.

2. If the von Neumann-Wigner interpretation is true, then only macrophenomenal properties necessitate collapse.

3. If macrophenomenal properties are constituted by only some combinations of micro(proto)phenomenal properties and they necessitate collapse, then only some combinations of micro(proto)phenomenal properties necessitate collapse.

4. If only some special combinations of micro(proto)phenomenal properties necessitate collapse, then collapse is strongly emergent.

5. Collapse as a fundamental process is not strongly emergent.

$\therefore 6$. If CRP is true, then the von Neumann-Wigner interpretation is false.

One might reject point 4 by arguing from a panprotopsychist perspective: collapse may be necessitated by any phenomenal properties, which, however, are constituted only by certain combinations of protophenomenal properties. Even if this is true, it does not render the argument invalid. In fact, Russellian panprotopsychism supports that microphysical truths are grounded in protophenomenal truths. Therefore, collapse, necessitated by only certain protophenomenal combinations, is arguably necessitated by the 
corresponding microphysical combinations. If one accepts that collapse is a physical process and that the physical world is causally closed, then being triggered only by certain microphysical combinations still makes collapse strongly emergent.

Objective collapse theories hold that wave function collapse results from the interaction between the microsystem and the measuring apparatus, irrespective of the presence of consciousness. They regard unitary evolution as an incomplete part of QM, supplemented by a non-unitary collapse process which is size-dependent. For microsystems, collapse does not occur (or occurs only occasionally), while for macrosystems such process occurs almost immediately.

If objective collapse theories are true, then an electron either never collapses or collapses at an extremely low rate. But the latter case indeed requires a preferred basis. This is already suspicious, as favouring a particular set of spin states - say spin along the z-axis - breaks the rotational symmetry. For the sake of the collapse theories, let's assume the electron is not that fundamental, and its internal mechanism is in charge of such symmetry breaking. At any rate, an electron collapses to a basis which depends primarily on its environment, say the apparatus and the observer. If we set up the apparatus to measure its spin along the x-axis, the electron would collapse to definite spin states along the $\mathrm{x}$-axis, even if it has a preferred basis along the $\mathrm{z}$-axis.

If microphysical truths are grounded in micro(proto)phenomenal truths, then arguably the micro(proto)phenomenal properties of an electron would collapse to the corresponding basis set dictated by its environment (under a spin measurement, say). Since nothing is fundamentally different between the electron and its environment (according to CRP they are both grounded in the same possible set of microphysical and microphenomenal truths), the dominance of the environment is arguably explained by its relatively large size. Now think about the whole world as a huge apparatus that performs a measurement on me. Even if our phenomenal properties collapse to our own preferred basis sufficiently frequently, it is possible for the environment-induced collapse to occur even more frequently. It is unclear why our phenomenal properties can only collapse to those definite states (e.g. |perceiving up $\rangle$ and |perceiving down $\rangle$ ), but not the superposed ones (e.g. 
$\frac{1}{\sqrt{2}}(\mid$ perceiving up $\rangle \pm \mid$ perceiving down $\left.\left.\rangle\right)\right)$ even under very different environments. To put this formally:

1. If CRP is true, then micro(proto)phenomenal properties are categorical bases for microphysical properties, and collectively they constitute macrophenomenal properties.

2. If collapse is objective, then a microsystem (e.g. an electron) collapses to a basis depending on its environment (e.g. apparatus and observer in a measurement).

3. If microphysical properties collapse to a basis depending on the environment, and their categorical bases are micro(proto)phenomenal properties, then micro(proto)phenomenal properties also collapse to a basis depending on the environment.

4. If micro(proto)phenomenal properties collapse to a basis depending on the environment, and they collectively constitute macrophenomenal properties, then the set of possible macrophenomenal properties (e.g. those that ground the physical states of |perceiving up $\rangle / \mid$ perceiving down $\rangle$ or $\frac{1}{\sqrt{2}}(\mid$ perceiving up $\rangle \pm \mid$ perceiving down $\left.\left.\rangle\right)\right)$ depends on the environment.

5. The set of possible macrophenomenal properties do not vary with the environment (e.g. one never perceive superposed states $\frac{1}{\sqrt{2}}(\mid$ perceiving up $\rangle \pm \mid$ perceiving down $\left.\left.\rangle\right)\right)$.

$\therefore 6$. If CRP is true, collapse is not objective.

Finally, let's consider the epistemic interpretation of wave function collapse, which holds that quantum states are ontological entities while their collapse is merely epistemic. Both hidden variable theories and the RS interpretation fall into this category. The previous incompatibility arguments, which attack either the epistemic picture of quantum states or the ontological picture of collapse, are inapplicable to them. Between hidden variable theories and the RS interpretation, the former seems to suffer from heavier ontological burden due to the introduction of hidden variables and their guiding laws. They add ontological complexity without making further detectable predictions, thus not a favourable option according to Occam's razor. On the other hand, the "let quantum be quantum" picture of the RS interpretation preserves the simplicity and elegance of QM.

Besides, hidden variable theories have to sacrifice locality for determinism as a result of Bell's theorem. The simplest version, Bohmian mechanics, introduces particle positions as 
hidden variables, as well as additional laws (e.g. the guiding equation and the quantum equilibrium hypothesis) merely to remove indeterminism. The hidden variables along with their laws are independent of the unitary evolution of wave function (which is now called "pilot wave"), and serve as extra theoretical constructions. If CRP is true, then micro(proto)phenomenal properties have to be structured in a more complex way in order to ground the two layers of microphysical properties, the pilot wave and the hidden variable. At any rate, the RS interpretation is more plausible in terms of what is required to construct the micro(proto)phenomenal properties.

Overall, accepting CRP rules out most options, leaving RS perhaps the most plausible interpretation. Because both physicalism and dualism suffer from severe objections, the conceivability/knowledge arguments for the former and the causal argument for the latter (see Chalmers 2015), CRP is perhaps the only viable option immune to objections from either side. Therefore, the analysis above provides support to the seemingly counterintuitive RS interpretation of QM.

\subsection{From RS to CRP}

Now we will reverse the direction of our argument to see if RS could offer some support to CRP. In fact, if RS is true, then the whole world is by nature quantum mechanical and governed by unitary evolution only. Classical phenomena emerge from interactions, say between a system and its environment, by mechanisms such as quantum Darwinism (see Zurek 2009).

First let's suppose phenomenal truths are wholly grounded in structural truths (I will explain later if it is not the case). Consider a conscious system, such as my brain. It consists of a large number of subsystems with quite complex interactions. Consciousness (or macrophenomenal properties) emerges not from some special spatial distribution, but instead from non-spatial relations between the subsystems (e.g. causal relations or bonding relations). One can imagine that a magical elf comes into my brain and re-arranges these subsystems spatially, while preserving their non-spatial relations. The same state of con- 
sciousness would arguably persist. This is to say, consciousness supervenes nomologically on the subsystems and the structure of their non-spatial relations.

If RS is true, then Hilbert space (plus the unitary maps written on it) is arguably the unique source of such structure. Between two Hilbert spaces, the main non-spatial structural difference lies in their dimensions. But merely a dimensional difference is probably incapable of explaining phenomenal properties being associated only with certain macrosystems. It is more plausible to associate phenomenal properties with any system, regardless whether it is a macrosystem or a microsystem. I recall that the whole argument, however, presupposes that the phenomenal truths are wholly grounded in structural truths. To put this formally:

1. If RS is true, then non-spatial structural truths are wholly grounded in dimensional truths.

2. If phenomenal truths are wholly grounded in structural truths, then they are wholly grounded in non-spatial structural truths.

3. If phenomenal truths are wholly grounded in non-spatial structural truths and the latter are wholly grounded in dimensional truths, then phenomenal truths are wholly grounded in dimensional truths.

4. If phenomenal truths are wholly grounded in dimensional truths, then they are not fundamentally different across systems.

5. If phenomenal truths are not fundamentally different across systems, then phenomenal properties are universal.

6. If phenomenal properties are universal, then panpsychism is true.

$\therefore 7$. If $\mathrm{RS}$ is true and phenomenal truths are wholly grounded in structural truths, then panpsychism is true.

Critics may reject point 1 by claiming that there are additional non-spatial structural truths that are not grounded in dimensional truths. It is plausible that physics describes all properties that are structural or dispositional. So these additional truths have to be realised in physical terms. Under the "let quantum be quantum" picture of the RS interpretation, the full physical picture of a system can be described simply by the 
unitary evolution of its quantum state (regarded as a vector in Hilbert space). The nonspatial structure is arguably either about the dimension of the complete Hilbert space that describes the whole system, or about the dimensions of its subspaces "travelled" by unitary evolution 11 Alternatively, critics may reject point 4 by arguing that phenomenal properties are strongly emergent upon certain dimensional properties. It is hard to see how this is possible. Perhaps there are critical dimensions above which phenomenal properties emerge. But still this is not convincing.

The argument above supports panpsychism, given that phenomenal truths are wholly grounded in structural truths. On the other hand, if phenomenal truths are not wholly grounded in structural truths, then they are also grounded in non-structural or categorical truths, which are by definition (proto)phenomenal. It is noted that any property of the conscious system is constituted by the properties of its subsystems along with their structures. Since (proto)phenomenal properties are not structural properties, in order to have them instantiated in the conscious system, they must already be instantiated in the constitutive subsystems. For a given conscious system, its subsystems are somewhat indefinite. We may in fact regard all the constitutive microsystems as such subsystems. This entails the instantiation of (proto)phenomenal properties in these microsystems, supporting a version of pan(proto)psychism. To put this formally,

1. If phenomenal truths are not wholly grounded in structural truths, then they are grounded in non-structural truths of subsystems that constitute the conscious system.

\footnotetext{
${ }^{1}$ In order to reduce the dimension of Hilbert space into a finite dimension, we need to discretise the physical space and assume consciousness is not lost after such discretisation. Now suppose a brain has a microphysical state in an N-dimensional Hilbert space. Given its Hamiltonian, an n-dimensional subspace is associated with consciousness, assuming that any superposition of conscious states is also a conscious state. Since conscious states remain conscious under unitary evolution, the conscious subspace has to be an invariant subspace. We may regard it as a spectral subspace of a binary-valued "consciousness observable", whatever it is, which commutes with the Hamiltonian. Maybe the consciousness observable is a function of other physical observables that commute with the Hamiltonian. At any rate, it is the only observable other than the Hamiltonian that is ever relevant to the structural explanation of consciousness. Note that commutativity is required in order to guarantee spectral subspaces of the observable to be invariant under unitary evolution. Apart from a unitary basis transform, the Hamiltonian and the consciousness observable can be fully recovered by the dimensions of their spectral subspaces, along with the corresponding eigenvalues. Since eigenvalues of the Hamiltonian provide energy levels which are not part of the structural information, structural truths relevant to consciousness may be represented solely by a list of dimensions $\left(n_{1}, m_{1}\right),\left(n_{1}, m_{2}\right), \cdots$. A pair $\left(n_{i}, m_{i}\right)$ contains structural truths about the $i$-th spectral subspace of the Hamiltonian (dimension $n_{i}+m_{i}$ reflects energy degeneracy), decomposed into an $n_{i}$-dimensional conscious subspace and an $m_{i}$-dimensional unconscious subspace.
} 
2. If phenomenal truths are grounded in non-structural truths of the constitutive subsystems, then they are grounded in (proto)phenomenal truths of the subsystems.

3. If phenomenal truths are grounded in (proto)phenomenal truths of the constitutive subsystems, then instantiation of phenomenal properties entails instantiation of (proto)phenomenal properties in the subsystems.

4. Phenomenal properties are instantiated in a conscious system constituted by a large number of microsystems.

5. If (proto)phenomenal properties are instantiated in the microsystems that constitute a conscious system, then pan(proto)psychism is true.

$\therefore 6$. If phenomenal truths are not wholly grounded in structural truths, pan(proto)psychism is true.

A possible objection to point 2 is that the non-structural truths involved are not (proto)phenomenal truths. Instead they are about the instantiation of dispositional properties or quiddities (i.e. categorical bases of physical dispositions). But the dispositions, such as mass or electric charge, are ultimately about relations and structures, and reveal themselves only in interactions. Therefore, their instantiation is rather specified in structural truths. On the other hand, quiddities as categorical properties are non-structural. It is plausible for the truths about their instantiation to ground phenomenal truths. But in this case, quiddities are also the categorical properties that constitute phenomenal properties, and thus are by definition (proto)phenomenal. Clearly this is a Russellian version of pan(proto)psychism.

Combining the two arguments shown above, one for phenomenal truths wholly grounded in structural truths and the other for phenomenal truths not wholly grounded in structural truths, we conclude that RS entails pan(proto)psychism. These arguments further shed some light to the type of pan(proto)psychism that RS supports. In fact, if phenomenal truths are not wholly grounded in structural truths, then they are also grounded in (proto)phenomenal truths of the constitutive microsystems. This supports a constitutive version of pan(proto)psychism. On the other hand, if phenomenal truths are wholly grounded in structural truths, then this suggests contingent laws that connect structural 
properties to phenomenal properties. We term this version of non-constitutive panpsychism structural panpsychism.

Utilising the symmetry between micro- and macrosystems provided by RS, we may further argue that RS supports a Russellian version of pan(proto)psychism. We get to know the spin state of an electron by coupling it to our brain state (via an apparatus). The electron and the brain collectively are in a superposed state of |up, perceiving up $\rangle$ and |down, perceiving down $\rangle$. The brain states, |perceiving up $\rangle$ and |perceiving down $\rangle$, are distinguished from the inside, i.e. from their distinct macrophenomenal properties. It is thereby plausible that the distinct macrophenomenal properties serve as the categorical bases for the distinct macrophysical properties of the two brain states (or observer states).

According to $\mathrm{RS}$, the electron has a definite spin state only relative to a definite observer state, say, |up $\rangle$ relative to |perceiving up $\rangle$. This relation is arguably symmetric, i.e. it is also reasonable to say that a definite observer state is only relative to a definite spin state of the electron. Since observer states can be distinguished categorically by macrophenomenal properties, spin states of the electron can be distinguished categorically as well, by their distinct micro(proto)phenomenal properties. Therefore, micro(proto)phenomenal properties ground microphysical properties categorically. The technical details are listed as follows:

1. If $\mathrm{RS}$ is true, then a measurement imposes a symmetric relation (a relative-to relation) between distinct microphysical states (of a microsystem) and distinct macrophysical states (of an observer).

2. Distinct phenomenal properties (e.g. perceiving up and perceiving down) are categorical bases for distinct macrophysical states (e.g. |perceiving up $\rangle$ and |perceiving down $\rangle$ ). 3. If distinct microphysical states (e.g. $|\mathrm{up}\rangle$ and $\mid$ down $\rangle)$ are relative to distinct macrophysical states (e.g. |perceiving up $\rangle$ and |perceiving down $\rangle$ ), and the latter are grounded categorically in distinct phenomenal properties (e.g. perceiving up and perceiving down), then distinct microphysical states are also relative to these phenomenal properties.

4. If distinct physical states of a microsystem are relative to distinct phenomenal properties of the observer, then inversely distinct physical states of the observer are relative 
to distinct (proto)phenomenal properties of the microsystem.

5. If distinct physical states of the observer are relative to distinct microsystem properties both physically (point 1) and (proto)phenomenally (point 4), then distinct physical properties of the microsystem are associated with distinct (proto)phenomenal properties.

6. If distinct microphysical properties are associated with distinct micro(proto)phenomenal properties, then the latter are the categorical bases for the former.

$\therefore 7$. If RS is true, then micro(proto)phenomenal properties of a microsystem are the categorical bases for its microphysical properties.

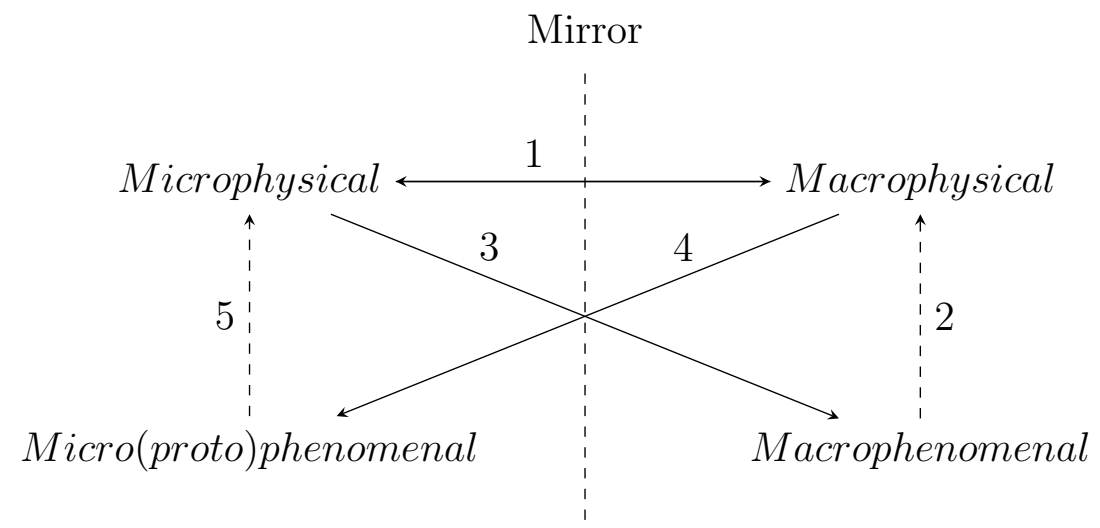

The argument, despite being complex, is conceptually clear. We can explain it schematically using the figure above. The dashed arrows denote the grounding relations and the solid arrows are the relative-to relations endorsed by the RS interpretation. These arrows are indexed according to their orders in the argument. The RS interpretation provides symmetry between micro- and macrosystems in a measurement, represented by the "mirror" placed in the middle. The argument starts from arrow 1 (rightwards) and arrow 2. Together they entail arrow 3. Arrow 3 is then mirrored to arrow 4, which combined with arrow 1 (leftwards) entails arrow 5 .

Some points of the argument may require further clarification. On point 4 , the measuring system which conventionally involves an apparatus and a conscious observer has nothing special. In fact, how do we define a "measurement" metaphysically? It is arguably just some definite arrangement that couples two originally separated systems. Under the RS interpretation, the two sides of a measurement are symmetric, in the sense that a definite physical state of one side is relative to a definite physical state of the other 
side and vice versa. Metaphysically, it does not matter which side measures and which side gets measured. Therefore in a measurement of, say, the spin state of an electron, we may swap the measuring side and the measured side. The relative-to relation between the phenomenal properties of the measuring side and the physical properties of the measured side should hold after the swap. Even if we take a panprotopsychist view, such that the electron, now regarded as the measuring side, only have protophenomenal properties, the relative-to relation is arguably still applicable. Otherwise, when certain protophenomenal properties combine to form phenomenal properties, the relative-to relation emerges. This implies that the relative-to relation emerges only on certain combinations of microphysical properties. The original relative-to relation, between the physical states of two systems, is thus contingent and strongly emergent. Since it is unlikely for such a fundamental and ubiquitous relation to be strongly emergent, the relative-to relation is arguably applicable for both phenomenal and protophenomenal properties.

On point 5 , the premise states that in a measurement defined above, two relative-to relations are established. The first one is according to point 1, which links the macrophysical properties of a macrosystem (e.g. |perceiving up $\rangle$ or |perceiving down $\rangle$ of the observer) to the microphysical properties of a microsystem (e.g. $|\mathrm{up}\rangle$ or $\mid$ down $\rangle$ of the electron). The second one is according to point 4, which links the same macrophysical properties to the categorical properties of the microsystem (e.g. what it is like to be in $|u p\rangle$ or |down $\rangle$, if we take a panpsychist view). The relative-to relation is arguably a one-to-one correspondence. The two relative-to relations thus establish a one-to-one correspondence between the microphysical properties (e.g. |up $\rangle$ or $\mid$ down $\rangle$ ) and the (proto)phenomenal properties (e.g. what it is like to be in $|\mathrm{up}\rangle$ or $\mid$ down $\rangle$ ). The latter, therefore, distinguish the former categorically. This rejects the non-Russellian view and suggests that some (proto)phenomenal properties are categorical bases for microphysical properties.

Overall, RS supports either constitutive Russellian pan(proto)psychism or structural Russellian panpsychism. The latter, though, cannot avoid objection from the causal argument. By suggesting that phenomenal properties are connected to structural properties only contingently (via some psychophysical laws), it leads to either epiphenomenalism 
or overdetermination. At any rate, RS rejects most of the options placed on the table, leaving CRP perhaps the most plausible solution to the hard problem of consciousness.

\section{Ontological Picture}

In this section we will discuss the theoretical burdens that RS and CRP have. Despite being potential solutions to different problems, they actually share pretty much the same theoretical requirement. We will see if combined together they could provide a unified ontological picture.

Perhaps the most counter-intuitive part about RS is proposed by the thought experiment of quantum suicide. In this thought experiment, Schrödinger's famous cat is replaced by an observer, say myself. Therefore, I am simultaneously the observed object and the observing subject. Critics of RS may therefore claim that RS entails immortal-

ity, for there is always a "branch" in which I survive. This reminds us of Parfit's (1984) thought experiment of teletransportation, which leads to the conclusion that personal identity can be indeterminate. It implies a somewhat deflationary account of subjects (i.e. subjects of experience), in the sense that they are not metaphysically primitive.

The same issue is faced by constitutive panpsychism, which suggests that microphenomenal properties constitute macrophenomenal properties. If macrosubjects (i.e. subjects of macrophenomenal experience) were primitive, then they could not be constructed by microsubjects (i.e. subjects of microphenomenal experience) due to the subject/subject gap (see Chalmers 2015). Even if we adopt the panprotopsychist view, it is still implausible, as combination of protophenomenal properties does not necessitate the existence of primitive subjects (the nonsubject/subject gap). Therefore, accepting CRP requires rejecting the view that subjects are metaphysically primitive.

The deflationary view of subjects, despite of being somewhat counter-intuitive, provides the basis for the viable solutions to both problems, the interpretation of $\mathrm{QM}$ and the hard problem of consciousness. Accepting this view is to feed two birds with one 
scone. Perhaps the only plausible option is to admit that subjects are derivative entities, constructed by bundles of experiences connected by memory. It is also plausible that subjects are ontologically dependent on a collection of causal chains (or some general set of relations), consistent with Kripke's (1972) causal account of reference.

We may provide more credibility to this deflationary account with the help of introspection. In introspection all we may find are a subject and a phenomenal quality paired by an awareness relation. They form an inseparable triplet, as Russell wrote in On Propositions (1919):

The act, or subject, is schematically convenient, but not empirically discoverable. It seems to serve the some sort of purpose as is served by points and instants, by numbers and particles and the rest of the apparatus of mathematics. All these things have to be constructed, not postulated: they are not stuff of the world, but assemblages which it is convenient to be able to designate as if they were single things.

Subject does not seem to be an introspective datum. Every time we think about it, it degrades to an object. Besides, awareness of being a subject is arguably grounded in the awareness of the subject's own phenomenal experiences, and perhaps in relation with external entities. One might object, however, that we are capable of introspecting the awareness relation alone. When looking into my sensation of red (i.e. the awareness of a red quality), I am aware of the fact that I sense the red quality, arguably a second-order awareness. It is actually the awareness of (a representation of) the mental state in which I sense the red quality. This mental state itself involves the whole triplet (I and the red quality connected by the red sensation). Therefore, a purely abstract and detached awareness relation does not seem empirically discoverable. Overall, we will assign the term "phenomenal property" to the whole inseparable triplet.

Phenomenal properties are commonly defined as properties characterising what it is like to be a conscious subject. But because accepting CRP and/or RS necessitates 
a deflationary account of subjects, such that subjects can be further reduced to, say, bundles of experiences. Therefore, the definition of phenomenal properties can be further reduced to topic-neutral terms (here we refer to terms not mentioning subjects explicitly). A viable option is to replace "subject" in the definition by "state" or "event", as Russell wrote in Analysis of Matter (1928):

A piece of matter is a logical structure composed of events; the causal laws of the events concerned, and the abstract logical properties of their spatio-temporal relations, are more or less known, but their intrinsic character is not known.

Therefore, we define phenomenal properties as properties characterising what it is like to be in a particular state (microstate for a microsystem or macrostate for a macrosystem). This is fully consistent with the RS interpretation. An observer who measures the spin state of an electron is, along with this electron, in a state superposed by |up, perceiving up $\rangle$ and |down, perceiving down $\rangle$. Defining phenomenal properties with regard to states instead of subjects naturally distinguishes the two phenomenal experiences (i.e. perceiving up and perceiving down) and hence the two outcomes (i.e. spin up and spin down).

Accepting both CRP and RS provides us with a simple and elegant ontological picture. Microphysical properties, defined as the properties that characterise microstates dispositionally from the outside, are grounded categorically in micro(proto)phenomenal properties, from the inside. They constitute macrophysical properties according to the laws of QM, which are the physical (or dispositional) aspects of the fundamental laws responsible for combining microstates into macrostates. Isomorphically, there are also phenomenal (or categorical) aspects of such laws for combining micro(proto)phenomenal properties into macrophenomenal properties. What we have direct access to (i.e. knowledge by acquaintance as defined in Russell 1910) are macrophenomenal properties characterising what it is like to be in a particular brain state.

But what about the preferred basis? For a measurement state superposed by |up, per- 
ceiving up $\rangle$ and |down, perceiving down $\rangle$, presumably only two distinct sets of macrophenomenal properties, perceiving up and perceiving down, are present. However, it is unclear why superposed consciousness cannot exist. There is a physical counterpart of this question: Why does the spin measurement result in either |up $\rangle$ or $\mid$ down $\rangle$ rather than the superposition? Quantum Darwinism proposed by Zurek (2009) provides a plausible answer: Unitary evolution alone can lead to a preferred basis as a result of environmentinduced superselection. Pointer states (e.g. $\mid$ up $\rangle$ and $\mid$ down $\rangle$ ) are those orthogonal states that, without being disturbed or destroyed, can be widely copied and disseminated by the environment. Superposed states, on the other hand, cannot leave sufficient copies in the environment. During a measurement all we can access are actually the plentiful descendants left in the environment. Effectively, the environment serves as an essential witness and communication channel for the microstate being measured.

The same mechanism may apply to macrophenomenal properties. The brain itself provides a plentiful environment, acting as a witness of the microstates involved. Due to quantum Darwinism, a macrostate is constituted by widely copied and disseminated microstates, as a superposition of, say, $\mid$ perceiving up $\rangle_{1} \mid$ perceiving up $\rangle_{2} \mid$ perceiving up $\rangle_{3} \ldots$ and $\mid$ perceiving down $\rangle_{1} \mid$ perceiving down $\rangle_{2} \mid$ perceiving down $\rangle_{3} \cdots$, where $1,2,3, \cdots$ are indices of subsystems in the brain. As a result, the subspace spanned by |perceiving up $\rangle$ and |perceiving down $\rangle$ has a natural basis consisting of orthogonal pointer states. These states are grounded categorically in distinct phenomenal properties, characterising what it is like to know the electron spins up and spins down respectively. This is consistent with the no-cloning theorem (see Wootters and Zurek 1982) which claims that it is only possible to clone a certain set of orthogonal quantum states (|perceiving up $\rangle$ and |perceiving down $\rangle$ are in this set while their superpositions are not).

As a final note, the interpretation puzzle about quantum mechanical probabilities may be mentioned here. Critics of RS sometimes argue that if distinct measurement outcomes are observed only by distinct subjects, then they should all have a probability of one. Surely the situation is a bit counter-intuitive, as we usually talk about probability only in the context of a single subject, which is presumably identical over time. 
However, we have already rejected this presumption by accepting a deflationary account of subjects. Indeed, all we have are distinct phenomenal properties that ground distinct physical properties categorically. Quantum mechanical probability can therefore be interpreted objectively, as the conditional probability of either certain physical properties or their grounding phenomenal properties. Such interpretation is within the context of the single quantum world (although it can have many classical "miniworlds"), or the "multiverse" (see Bousso and Susskind 2012). For example, the probability of $|u p\rangle$ may be interpreted as the probability of instantiation of the perceiving-up phenomenal properties after the measurement, conditional to the physical and phenomenal properties before the measurement.

\section{The Combination Problem}

Pan(proto)psychism, especially constitutive pan(proto)psychism, faces a major challenge of the combination problem: How do micro(proto)phenomenal properties constitute the kind of macrophenomenal properties that we are familiar with? It was first proposed by William James in The Principles of Psychology (1895):

Where the elemental units are supposed to be feelings, the case is in no wise altered. Take a hundred of them, shuffle them and pack them as close together as you can (whatever that may mean); still each remains the same feeling it always was, shut in its own skin, windowless, ignorant of what the other feelings are and mean. There would be a hundred-and-first feeling there, if, when a group or series of such feelings were set up, a consciousness belonging to the group as such should emerge. And this 101st feeling would be a totally new fact; the 100 original feelings might, by a curious physical law, be a signal for its creation, when they came together; but they would have no substantial identity with it, nor it with them, and one could never deduce the one from the others, or (in any intelligible sense) say that they evolved it. 
James seems to argue that phenomenal properties cannot aggregate into further phenomenal properties. However, this intuition about property combination is arguably grounded in our intuition about classical physics. In QM it is plausible that combining distinct properties brings in extra information.

Let's assign a property to an electron. For example, we can assign a definite value (up or down) to its spin, which is regarded as a random variable denoted by $S$. The amount of information that is introduced by this assignment is measured by the mutual information: $I(\mathcal{A} ; S)=H(\mathcal{A})+H(S)-H(\mathcal{A}, S)$ in which $\mathcal{A}$ is the state of the electron with an unspecified spin. If the electron were a classical object, $H$ would be (joint) Shannon entropy that follows $H(\mathcal{A}) \leq H(\mathcal{A}, S)$ (see Cover and Thomas 2012). Therefore, the information introduced by the spin property, $I(\mathcal{A} ; S)$, can never exceed $H(S)$, which is the amount of information carried by the spin property alone. But in reality the electron is quantum-mechanical. So Shannon entropy needs to be replaced by Von Neumann entropy, which no longer follows the inequality. In an extreme case, the electron stays in a pure state $|a, \mathrm{up}\rangle+\mid b$,down $\rangle$. This implies $H(\mathcal{A}, S)=0$ but $H(\mathcal{A})>0$, for properties other than the spin can only be described probabilistically by a density matrix.

The example above shows that in a quantum system, a property can bring in more information than that contained in the property alone. If we accept that the brain is a macroscopic quantum system (indeed it is, according to RS which holds QM is universal), then it is not implausible that combining phenomenal properties reveals extra phenomenal properties. James's argument arises from the intuition about our living world, which can be explained by classical physics sufficiently well. Such intuition might not apply to the macrophenomenal properties that characterise the macrostates of our brains, which are quantum-mechanical by their very nature.

\subsection{The subject combination problem}

James further argued in The Principles of Psychology (1895): 
Take a sentence of a dozen words, and take twelve men and tell to each one word. Then stand the men in a row or jam them in a bunch, and let each think of this word as intently as he will; nowhere will there be a consciousness of the whole sentence. We talk of the 'spirit of the age,' and the 'sentiment of the people,' and in various ways we hypostatize 'public opinion.' But we know this to be symbolic speech, and never dream that the spirit, opinion, sentiment, etc., constitute a consciousness other than, and additional to, that of the several individuals whom the words 'age,' 'people,' or 'public' denote. The private minds do not agglomerate into a higher compound mind.

This point is pretty clear. If the phenomenal properties of individuals cannot aggregate into some sort of "collective" phenomenal properties, then how could the micro(proto)phenomenal properties of particles aggregate into the macrophenomenal properties of our own? Given that we already take a deflationary account of subjects, a co-consciousness relation (see Chalmers 2017) seems to be sufficient for the explanatory purpose. It is plausible that the co-consciousness relation applies to phenomenal properties associated with one individual, but fails to apply to those associated with different individuals.

But what exactly is this co-consciousness relation? If micro(proto)phenomenal properties ground microphysical properties, then arguably such relations applied to micro(proto)phenomenal properties ground certain relations in the physical world. We do have a good candidate in a pure quantum world. According to quantum Darwinism, the environment serves as a communication channel that clones and disseminates information about pointer states. It is a lossy channel in the sense that only classical information (i.e. information about pointer states) gets transferred. Full (or a large extent of) quantum information, on the other hand, cannot be retrieved, unless we can access the full (or most of the) environment and the numerous copies left in the environment.

This mechanism establishes a co-accessibility relation in the physical world: Two sets of microphysical properties that fully characterise two microsystems are co-accessible if 
and only if the quantum information of either can be (almost) fully retrieved in the environment (or neighbourhood) of the other. Take the brain system as an example. For two distinct particles inside the same brain (or its essential subsystem responsible for consciousness), it is in principle possible that the information about the quantum state of one particle can be fully (or to a large extent) retrieved from the environment of the other. In this case, the microphysical properties of the two particles are coaccessible. This, of course, relies on the boundary of the environment. We may regard co-accessibility as being relative to the macrophenomenal properties under consideration. Since macrophenomenal properties characterise the state of a macrosystem categorically, the boundary of the environment is arguably the boundary of the macrosystem, which is well-defined.

The co-consciousness relation between two micro(proto)phenomenal properties is what grounds the co-accessibility relation of the corresponding microphysical properties. If two microsystems are connected by a classical communication channel, then the micro(proto)phenomenal properties that ground the respective microphysical properties are not co-conscious. Between two macrophenomenal properties, co-consciousness can be constructed by aggregating the co-consciousness relations between their constitutive micro(proto)phenomenal properties. Here the classical communication channel is a communication channel that has a lossy bottleneck, such that a large proportion of copies (of quantum states) are dissipated, incapable of being collected again. For instance, communication between two individual brains is mediated by their bodies and the surrounding physical world. Much of the copies originating from one brain are thus dissipated, rather than being collected by the other brain.

Let's conduct this analysis in more detail. The macrophenomenal properties of two individuals are the properties that categorically characterise their brain states. They correspond to two disjoint macrosystems, either their brains or some essential subsystems of their brains. Due to the classical communication channel between them, microphysical properties in one macrosystem are not fully accessible in the other macrosystem. This lack of co-accessibility is grounded in the fact that the micro(proto)phenomenal properties 
which ground the microphysical properties are not co-conscious. Constituted by these microphenomenal properties, macrophenomenal properties of the two individuals are not co-conscious.

In general, physical properties of other brains can only reach us via classical communication channels, thus only the classical information (about half of the full quantum information, measured by mutual information as in Zurek 2009) are directly accessible. Even if we could obtain the full knowledge about a quantum state from measurements, it is, in fact, inferred from the classical information recorded by the apparatus. The co-consciousness relation by its nature is absent between the phenomenal properties of two brains, as Russell commented in Analysis of Matter (1928):

The usual view would be that by psychology we acquire knowledge of our 'minds,' but that the only way to acquire knowledge of our brains is to have them examined by a physiologist, usually after we are dead, which seems somewhat unsatisfactory. I should say that what the physiologist sees when he looks at a brain is part of his own brain, not part of the brain he is examining.

On the other hand, our own brain states are what we have direct access to. The brain itself serves as the environment and the witness of its constitutive microphysical properties. Since it (or some part of it) is directly accessible, the full information (or at least more than the classical information) about the microphysical properties is also directly accessible. Any two macrophenomenal properties that characterise the same brain state are constituted by their respective microphenomenal properties, which are co-conscious, for the grounded microphysical properties are co-accessible within the boundary of the same macrosystem (the brain or its essential part). Constituted by co-conscious microphenomenal properties, these macrophenomenal properties themselves are co-conscious. In conclusion, there is always a co-consciousness relation between macrophenomenal properties that characterise the same brain state. Together with the analysis given in previous paragraphs, we have a plausible explanation of the fact that phenomenal properties are private knowledge, which can only be revealed from a first-person perspective. 


\subsection{The quality combination problem}

The quality combination problem concerns the combination rules of the qualities involved in micro(proto)phenomenal properties. The major objection comes from the palette problem, which argues that the limited qualities of micro(proto)phenomenal properties cannot combine into the plentiful qualities of macrophenomenal properties.

Despite that macrophenomenal properties presumably deal with pointer states only, e.g. either perceiving up or perceiving down but not their superpositions, micro(proto)phenomenal properties are not limited by this. According to quantum Darwinism, objective existence in its classical sense, i.e. an object appearing only in one of its pointer states, is merely due to the environment playing a role of witness. Micro(proto)phenomenal properties, on the other hand, concern the microsystem itself, irrespective of the environment. An electron can therefore have micro(proto)phenomenal properties associated with states of definite spins along the z-axis, as well as states of definite spins along the $\mathrm{x}$-axis, despite the fact that the environment cannot record both.

It is therefore plausible that even a single electron can have rich qualities of micro(proto)phenomenal properties. Our intuition about a microscopic object such as an electron is formed by our direct observation. But what we actually observe are the numerous copies of its pointer states left in the environment, not the object itself. Therefore, only the pointer states are directly perceivable. Micro(proto)phenomenal properties, on the other hand, ground microphysical properties of the object alone. There are arguably distinct micro(proto)phenomenal properties associated with every state in the Hilbert space, not merely the pointer states.

The palette problem does not seem to be an issue. But we need to take a step further and ask how these different qualities actually combine. Chalmers commented in The Combination Problem for Panpsychism (2017):

If the same entity simultaneously is aware of a degree of redness and aware of a degree of whiteness (at the same location), it is plausibly aware of pinkness 
(at that location). But in general separately instantiated qualities (the redness and whiteness of distinct objects) do not yield a combined quality, and nor do separately instantiated phenomenal qualities.

This indeed imposes a restriction on the nature of the combination rules. It is arguably related to the structure combination problem (see Sec 4.3), which asks for an explanation of the spatial structure of our visual fields. For now, let's suppose this phenomenal spatial structure is given. As Chalmers suggested, a phenomenal quality can combine with those in its neighbourhood (in the visual field), but not with those that are further away. Perhaps the co-consciousness relation proposed in Sec 4.1 needs to be further enriched by a metric (or at least topological) structure.

As a binary relation between phenomenal properties, co-consciousness guarantees the two connected phenomenal qualities can be experienced simultaneously. It does not prevent us from further exploring a stronger version though, such that the two connected phenomenal qualities are not only experienced simultaneously, but also combine into one uniform quality. It is plausible that the co-consciousness relation is structured hierarchically. For example, among all the co-conscious pairs of phenomenal properties, some may be bonded even more closely. We select these pairs and level up their pairing relations to a "level-1 co-consciousness relation". We may continue to apply the selection process to the set of all level- 1 co-conscious pairs in order to get a level-2 co-consciousness relation, etc. By repeating this procedure, we end up with a hierarchical structure of co-consciousness relations.

The hierarchical structure of co-consciousness relations presumably grounds an isomorphic structure of co-accessibility relations between physical properties, such that a subset of co-accessible pairs can have stronger dependence or bonding. As illustrated in the beginning of Sec 4, these pairs may have smaller von Neumann joint entropies, resulting in higher mutual information. This means that the pairs yield more information on combination, a result of quantum entanglement. Alternatively, recall that on defining the co-accessibility relation in Sec 4.1, we mentioned that the relation is only relative, in 
the sense that it does depend on the boundary of the system under consideration. We may therefore divide the whole brain into subsystems, such that the microphysical properties within one subsystem are co-conscious not only relative to the whole brain, but also relative to this subsystem. This serves as the physical basis of the level-1 co-accessibility relation, whose categorical basis is the level-1 co-consciousness relation. Within each of the subsystems, we may perform further division to obtain the level-2 co-accessibility relation, level-3 co-accessibility relation, etc.

\subsection{The structure combination problem}

This version of combination problem concerns the mismatch between the structure of macrophenomenal properties and the structure of macrophysical properties (say the structure observed when examining someone else's brain). As argued in Sec 2.2, it is not the spatial structure that matters, but the structure of some abstract relations (e.g. causal or co-consciousness relations). If quantum mechanics is universal, then such structure derives arguably from the structure of Hilbert space. Since Hilbert space has a linear and homogeneous structure, it is capable of providing a smooth and homogeneous structure for the phenomenal properties.

An especially important example is the spatial structure of the visual and auditory fields that we perceive. We have seen in Sec 4.2 that different levels of co-consciousness relations can be established, perhaps by dividing the brain into less dependent (or less entangled) subsystems hierarchically. In correspondence to this, Hilbert space of the whole brain is decomposed into factors hierarchically, such that the brain state, as a vector in Hilbert space, contains strongly entangled components within a factor space while less entangled components in different factors. This provides a basis for the phenomenal spatial structure, for the hierarchical structure of factors is isomorphic to the hierarchical structure of point sets in the phenomenal space. In this way, co-consciousness relations supply a topology for the phenomenal space.

Co-consciousness relation therefore serves as a key component of solving the phe- 
nomenal binding problem. Its hierarchical structure grounds the phenomenal spatial structure. Since co-consciousness grounds co-accessibility between physical properties, this hierarchical structure should manifest itself in the physical world as well. This implies that the physical spatial structure is emergent too, from the hierarchical structure of co-accessibility relations and ultimately quantum information. Indeed, this is a promising account of physical space endorsed by Cao and Carroll (2018), suggesting that the Euclidean metric of the physical space derives from quantum mutual information (defined in the beginning of Sec 4). We can conclude that the similarity between phenomenal spatial structure and physical spatial structure is at least partly due to the similar mechanisms responsible for their emergence. Both spatial structures emerge from quantum information, in the form of the hierarchical co-consciousness/co-accessibility relations. Utilising quantum mechanics therefore resolves the structural mismatch between the physical and the phenomenal.

Can we say anything more about the relation between the physical and the phenomenal? Because both have isomorphic structures grounded in co-consciousness/coaccessibility relations, we might further speculate that the whole quantum cosmos is conscious. The physical space simply emerges from the co-consciousness relations of this giant conscious subject. This is, however, a wild speculation and I am not positioned to claim it is plausible. At any rate, quantum information plays a role in the constitution of the physical world as well as our own consciousness in the form of co-consciousness/coaccessibility relations. It is, therefore, completely natural to have a certain degree of structural similarity between the physical world and our own consciousness. This ought to be appreciated, because only by this similarity can we have a prior concept of spacetime and the capacity of understanding the physical world so well.

\subsection{The report combination problem}

This version of the combination problem is associated with the metaproblem of consciousness regarding how we report our phenomenal truths (see Chalmers 2018). The problem 
may be formulated as how the micro(proto)phenomenal properties combine to result in reports about consciousness.

In fact, if the reports about consciousness could be explained solely by topic-neutral terms that do not mention consciousness itself (or anything grounded in phenomenal truths), then our intuition about consciousness could be false and consciousness could be just an illusion. However, it is probably true that such a topic-neutral explanation can never be achieved, as the reports about consciousness are indeed grounded in phenomenal truths. This is consistent with, and perhaps entailed by, the RS interpretation of QM.

Consider our old friend, the measurement of an electron's spin state. If we hold a illusionist view of consciousness, we would probably represent the whole system by a superposition of |up, reporting up $\rangle$ and |down, reporting down $\rangle$. We use "reporting" instead of "perceiving" because "perceiving up" (or "perceiving down") was used to designate a set of phenomenal properties. Since we now take a illusionist view, there is no such thing as "perceiving" in its phenomenal sense, and we may describe the system only in pure physical terms. But then how do we explain the fact that only one outcome gets reported, or at least we only "feel" one such report? Illusionism, or at least its strong version, denies there is something it is like to be in a certain state (e.g. perceiving up or perceiving down). Ontologically, the two reports or outcomes are equally real. It is therefore not clear why we only "feel" one outcome, rather than both or their superposition.

If we admit that our own consciousness is real, then according to Sec 2.2, RS entails pan(proto)psychism. We now face the report combination problem. Our strategy is to show that reports about consciousness are indeed grounded in phenomenal truths. In fact, the "branching" of the two spin states in the measurement is arguably grounded in the mutual exclusiveness of the underlying phenomenal properties. That is to say, there lacks a co-consciousness relation between the two phenomenal properties (i.e. perceiving up and perceiving down), and the apparent branching of the measurement outcomes is grounded in this absence of co-consciousness. We may formulate this argument in the following way: 
1. If $\mathrm{RS}$ is true, then the whole measurement system can be in superposition.

2. On a superposed measurement state, all alternative reports exist ontologically.

3. In any measurement, it is only possible to report a definite outcome.

4. If all reports exist ontologically while only a definite outcome is "actually" reported, then there has to be an epistemic branching of alternative reports.

5. Epistemic branching between reports (reporting up and reporting down) is grounded in the lack of co-consciousness between perceptions (perceiving up and perceiving down). 6. If report of perception is not grounded in perception, any relation between alternative reports is not grounded in relations between perceptions.

$\therefore 7$. If $\mathrm{RS}$ is true, then the report of perception is grounded in perception.

One might reject point 5 by resorting to certain co-accessibility relations between physical properties, claiming that the apparent branching is grounded in physical relations instead of phenomenal relations. However, according to Sec 2.2, RS entails the Russellian version of pan(proto)psychism. This means that the co-accessibility relation itself is grounded in certain relation between phenomenal properties. Therefore, the apparent branching is still grounded in some phenomenal relation. Whatever it is, the argument continues to hold.

"Perception" in the argument above may be replaced by any phenomenal terms, such as feeling, experience, or more generally, state of consciousness. So how do we respond to the report combination problem? We have shown that reports about macrophenomenal truths are indeed grounded in macrophenomenal truths. Also there are not many conceptual obstacles to the combination of micro(proto)phenomenal properties into macrophenomenal properties, for in the previous sections we have already proposed plausible solutions to other combination problems. Putting them together, we get the proposition that reports about macrophenomenal truths are grounded in micro(proto)phenomenal truths. The combination of micro(proto)phenomenal properties, which forms the corresponding macrophenomenal properties, also plays a central role in reporting such phenomenal properties. Co-consciousness relations between micro(proto)phenomenal properties, which add up to the co-consciousness relation between macrophenomenal properties, are 
causally responsible for the apparent branching of their reports.

\section{Concluding Remarks}

If we can accept the deflationary account of subjects, then both CRP and RS are permissible. In particular, together they provide us with a unified ontological picture that may solve the combination problem. The conceivability of the panpsychist zombie is arguably rooted in our prior knowledge about subjects (e.g. separation between the quality/object and the awareness/subject). But if such knowledge is not justified, the conceivability argument no longer holds. In fact, the essence of a subject is arguably constituted by a bundle of co-conscious phenomenal properties. It therefore rigidly designates the same bundle of phenomenal properties in every world (actual or counterfactual), and connects to the reference or name of the biological entity only contingently. At any rate, having the same micro(proto)phenomenal (and microphysical) properties but different or non-existent macrophenomenal properties is inconceivable. Besides, macrophenomenal properties are not subject to the causal argument, for they are constituted by micro(proto)phenomenal properties which ground physical properties categorically. We may conclude that the ontological picture proposed here is immune to the argument from either side (i.e. the conceivability argument or the causal argument).

Apart from sharing the same requirement of deflationary subjects, CRP and RS both realise symmetry between micro- and macrosystems. They are mutually supportive, thus providing a new perspective of solving either the hard problem of consciousness or the interpretation puzzle of QM. More important, RS provides feasible options to solve the combination problem, which seems to be the most severe obstacle to CRP. Overall, I think there is sufficient credibility for us to at least consider the combination of CRP and RS as one of the plausible options for explaining the very nature of our consciousness and our world. To summarise our current position, we list some of the main propositions as follows:

1. If rejecting CRP, we would suffer from either the conceivability/knowledge argument 
or the causality argument on explaining consciousness.

2. Accepting CRP makes RS perhaps the most plausible interpretation of QM.

3. Accepting RS supports CRP, and provides viable solutions to different versions of the combination problem faced by CRP.

$\therefore 4$. It is perhaps plausible to accept both CRP and RS.

\section{References}

Bousso, R. and Susskind, L. 2012. 'Multiverse interpretation of quantum mechanics', Physical Review D 85(4), 045007.

Cao, C. and Carroll, S. M. 2018. 'Bulk entanglement gravity without a boundary: Towards finding einstein's equation in hilbert space', Physical Review D 97(8), 086003.

Chalmers, D. 2015. 'Panpsychism and panprotopsychism', Consciousness in the physical world: Perspectives on Russellian monism pp. 246-276.

Chalmers, D. J. 2017. 'The combination problem for panpsychism', Panpsychism: Contemporary Perspectives pp. 179-215.

Chalmers, D. J. 2018. 'The meta-problem of consciousness', Journal of Consciousness Studies 25(9-10), 6-61.

Cover, T. M. and Thomas, J. A. 2012. Elements of information theory, John Wiley \& Sons.

James, W. 1895. The principles of psychology, Henry Holt.

Kripke, S. A. 1972. Naming and necessity, in 'Semantics of natural language', Springer, pp. 253-355.

Parfit, D. 1984. Reasons and persons, OUP Oxford.

Russell, B. 1910. Knowledge by acquaintance and knowledge by description, in 'Proceedings of the Aristotelian Society', Vol. 11, JSTOR, pp. 108-128. 
Russell, B. 1919. 'On propositions: What they are and how they mean', Proceedings of the Aristotelian Society, Supplementary Volumes 2, 1-43.

Russell, B. 1928. The Analysis of Matter, Vol. 3, Kluwer Academic Publishers.

Wootters, W. K. and Zurek, W. H. 1982. 'A single quantum cannot be cloned', Nature $\mathbf{2 9 9}(5886)$, 802-803.

Zurek, W. H. 2009. 'Quantum darwinism', Nature physics 5(3), 181-188. 